\title{
Introduction of Mesenchymal Stem Cells for Liver Surgery (Hepatectomy and Transplantation)
}

\author{
Shinji Uemoto, Yasuhiro Fujimoto, Takumi Teratani, Hiroyuki Kanazawa, \\ Junji Iwasaki, Zhao Xiangdong, Yuki Masano, Shintaro Yagi, Koichiro Hata, \\ and Eiji Kobayashi
}

\begin{abstract}
In liver transplantation, prolonged ischemia and/or a relatively small graft (living, split, reduced) are the risk factors for liver dysfunction. Novel measures to enhance liver function with a smaller graft can be a clue for safe partial or living-donor liver transplantation or safe hepatectomy for malignant disease. The therapeutic potential and immunomodulatory effects of mesenchymal stem cells (MSCs) have been reported. In this chapter, recent finding on the positive effect of MSCs for liver transplantation and hepatectomy are discussed.

Our rat experiment revealed that introduction of MSCs provides trophic support to the I/R-injured liver by inhibiting hepatocellular apoptosis and by stimulating regeneration, which is shown with the pig model as well. In the rat liver transplantation model, portal transfusion of the MSCs ameliorates the injury of the liver graft after prolonged cold preservation and transplantation. Those findings together suggest a potential advantage with partial or living-donor liver transplantation. The most severe complication with cell therapy is embolus formation due to cell aggre-
\end{abstract}

\footnotetext{
S. Uemoto, M.D., Ph.D. ( $\square)$

Professor, Division of HBP Surgery and Transplantation,

Department of Surgery, Kyoto University Graduate School of Medicine,

54 Kawara-cho, Shogoin, Sakyo, Kyoto 606-8507, Japan

e-mail: uemoto@kuhp.kyoto-u.ac.jp

Y. Fujimoto, M.D., Ph.D.

Division of HBP Surgery and Transplantation, Department of Surgery, Kyoto University Graduate School of Medicine, 54 Kawara-cho, Shogoin, Sakyo, Kyoto 606-8507, Japan

Division of HBP Surgery, Department of Surgery, Shizuoka City Shizuoka Hospital, 10-93

Ote-machi, Aoi-ku, Shizuoka City, Shizuoka 420-8630, Japan

T. Teratani, Ph.D. • E. Kobayashi, M.D., Ph.D.

Division of Development Advanced Therapy, Center for Development of Advanced Medical Technology, Jichi Medical University,

3311-1, Yakushiji, Shimotsuke, Tochigi 329-0498, Japan

H. Kanazawa, M.D., Ph.D. • J. Iwasaki, M.D., Ph.D. • Z. Xiangdong, M.D., Ph.D.

Y. Masano, M.D. • S. Yagi, M.D., Ph.D. • K. Hata, M.D., Ph.D.

Division of HBP Surgery and Transplantation, Department of Surgery, Kyoto University

Graduate School of Medicine, 54 Kawara-cho, Shogoin, Sakyo, Kyoto 606-8507, Japan
}

K. Nakao et al. (eds.), Innovative Medicine, DOI 10.1007/978-4-431-55651-0_23 
gation. However, with modification of the solution, we can keep cells in a suspended form for several hours, which secures safe administration of MSCs.

Keywords Liver transplantation - Mesenchymal stem cell • Hepatectomy • Ischemia $\bullet$ Reperfusion $\bullet$ Liver failure $\bullet$ Living donor $\bullet$ Partial liver transplantation

\section{Clinical Liver Transplantation: Deceased Donor vs. Living Donor}

\section{Statistics}

Liver transplantation has been successfully used as a treatment for end-stage liver disease, though there has been discussion regarding where the liver graft is obtained, i.e., from a deceased donor or a living donor. In the USA, the majority of liver transplantation is done with deceased donors, and the number of living-donor liver transplantations (LDLTs) is limited. The reason why LDLT is not popular in the USA is due to the death of a donor in 2002. Since 2001, the number of patients who have undergone LDLT has declined. On the other hand, in Japan, most liver transplantation is done with LDLT. Though the organ transplantation law took effect in 1997, the number of deceased donor liver transplantations (DDLTs) has been minimal; this is partly because the law itself is very strict in comparison with those of Western countries. Even since modification of the law in 2010, the annual number of DDLTs has been limited to below 50. The ratio of the numbers of LDLTs to DDLTs is $10: 1$ [1].

\section{Operations and the Post-transplant Course}

In DDLT, the whole liver is used, and it has a high reserve capacity (Fig. 1). In other words, the liver volume is sufficient for the recipient throughout the postoperative course from the immediate post-transplant period. That is true with LDLT as well; therefore, a larger graft is preferable in terms of the recipient's postoperative course. However, harvesting a larger graft from a living donor may cause complications, and there have been some reports of donor deaths. Currently, we primarily use smaller grafts such as left lobe grafts, in our program, though small-for-size syndrome (SFSS) occasionally follows in recipients, which can be lethal. Since LDLT is a type of partial liver transplantation (PLTx), the possibility of SFSS is unavoidable. Functional impairment conditions such as prolonged cholestasis, ascites, coagulopathy, and encephalopathy are considered to result from SFSS that occurs after LDLT [2]. They strongly affect patient survival and clinical costs for the whole treatment. Therefore, novel measures to enhance liver function with smaller grafts may be the key to safe PLTx or LDLT. 


\section{Postoperative course (human): Whole vs. Partial LTX}
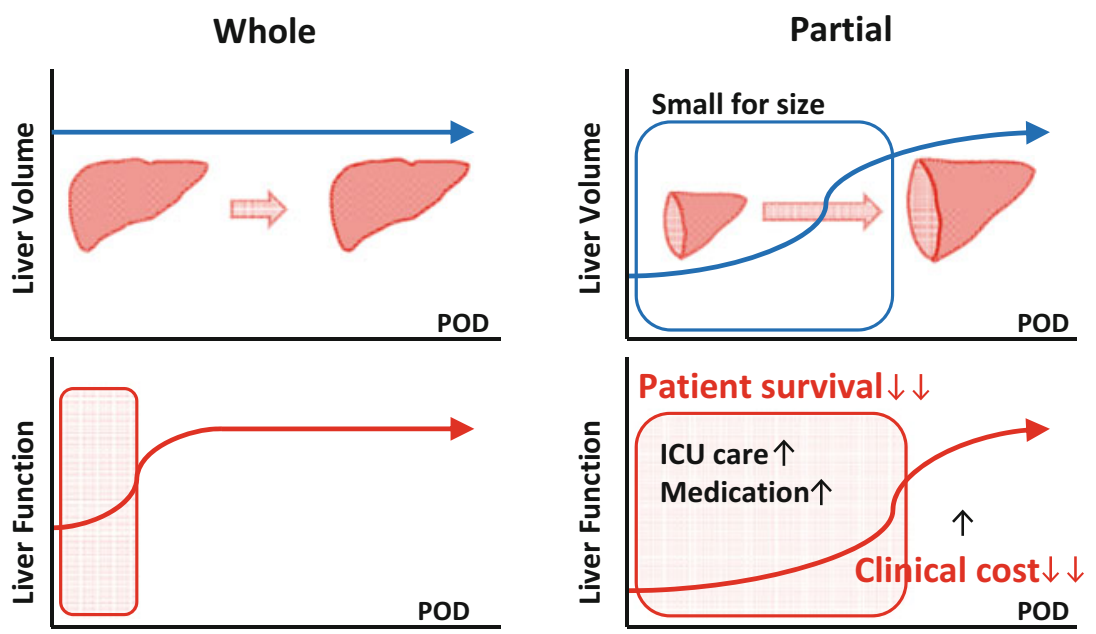

Fig. 1 Postoperative course of whole vs. partial liver transplantation, showing the difference between DDLT and LDLT after the operation. There is no change in size with DDLT, whereas graft enlargement is observed with LDLT. Functional recovery follows volume recovery; therefore, there should be some critical period with LDLT after operation

\section{Mesenchymal Stem Cells}

\section{Characteristics}

Mesenchymal stem cells (MSCs), present in the human body in large amounts, can be candidates for stem cell-based therapy for liver diseases. Recently, the therapeutic potential and immunomodulatory effect of MSCs have been revealed. Adipose tissue-derived mesenchymal stem cells (AT-MSCs), because of their high accessibility with minimal invasiveness, are especially attractive in the context of future clinical applications. MSCs can differentiate into multiple types of lineages, such as chondrogenic, osteogenic, and adipogenic, depending on the microenvironment around them. Importantly, human MSCs represent an advantageous cell type for allogeneic transplantation, as they are immunoprivileged with low human leukocyte antigen (HLA) I and no HLA II expression, thereby reducing the risk of allogeneic transplant rejection and preventing graft-versus-host disease (GVHD). According to Banas et al., adipose tissue-derived (AT) MSCs can improve liver functions, which we have verified by changes in the levels of biochemical parameters. Ammonia, uric acid, glutamic-pyruvic transaminase, and glutamic-oxaloacetic transaminase concentrations returned to a nearly normal level after AT-MSC transplantation. It was shown that AT-MSCs secrete interleukin 1 receptor (IL-1R), IL-6, 
IL-8, granulocyte colony-stimulating factor (G-CSF), granulocyte-macrophage colony-stimulating factor (GM-CSF), monocyte chemotactic protein 1, nerve growth factor, and hepatocyte growth factor, suggesting that AT-MSCs account for broad therapeutic efficacy in animal models of liver diseases and in the clinical settings of liver disease treatment [3].

\section{Isolation Techniques}

AT-MSCs can be derived from subcutaneous adipose tissue. Adipose tissue was minced with scissors and scalpels into less than $3 \mathrm{~mm}$ pieces and, after gentle shaking with an equal volume of $\operatorname{PBS}(-)$, the mixture was separated into two phases. The upper phase (containing stem cells, adipocytes, and blood), after washing with phosphate-buffered saline (PBS) (-), was enzymatically dissociated with $0.075 \%$ collagenase (type I)/PBS(-) for $1 \mathrm{~h}$ at $37^{\circ} \mathrm{C}$ with gentle shaking. The dissociated tissue was then mixed with an equal volume of DMEM, supplemented with $10 \%$ fetal bovine serum (FBS) and incubated for $10 \mathrm{~min}$ at room temperature. The solution was then separated into two phases. The lower phase was centrifuged at $1500 \mathrm{rpm}$ for $5 \mathrm{~min}$ at $20^{\circ} \mathrm{C}$. The cellular pellet was resuspended in $160 \mathrm{mM} \mathrm{NH}_{4} \mathrm{Cl}$ to eliminate erythrocytes and passed through a $40 \mu \mathrm{m}$ mesh filter into a new tube. The cells were resuspended in an equal volume of DMEM/10 \% FBS and then centrifuged. Isolation resulted in obtaining $7.7 \times 10^{6}$ of adherent cells for a primary culture from $5 \mathrm{~g}$ of adipose tissue (approximately $1.0 \times 10^{5}$ to $4.6 \times 10^{6} / 1 \mathrm{~g}$ ) after $7-10$ days of culture. The cells were suspended in DMEM/10 \% FBS plated in a concentration of $1-5 \times 10^{6}$ cells $/ 75 \mathrm{~cm}^{2}$. The cells with $70-80 \%$ confluence were harvested with $0.25 \%$ trypsin EDTA and then re-plated at $1.0 \times 10^{5}$ cells $/ 60 \mathrm{~mm}$ dish and used for analysis [4].

\section{Mesenchymal Stem Cells for Rat Hepatectomy}

\section{Rat Hepatectomy Model}

Our rat model provided the ideal animal model to research I/R injury after major hepatectomy by the Pringle maneuver or liver transplantation with small-for-size grafts (Fig. 2) [5]. Intestinal congestion was avoided during the application of hepatic ischemia by bypassing portal flow through nonischemic lobes (the right and caudate lobes). Moreover, the left lateral and the left portion of the medial lobes, as well as the nonischemic lobes, were excised at the onset of reflow to mimic the clinical condition of liver transplantation with small-for-size grafts or major hepatectomy by the Pringle maneuver in order to research postischemic liver functions. 


\section{Hepatectomy (70\%) with I/R injury model (rat)}

Lewis-rats, male

Warm ischemic time :40 min

Hepatectomy $70 \%$

MSC: Lewis-Rat, BM-MSC (passage 6 - 8)

MSC group $(n=6): 1 \times 10^{6}$ cells/body

Control group $(n=6)$ :PBS

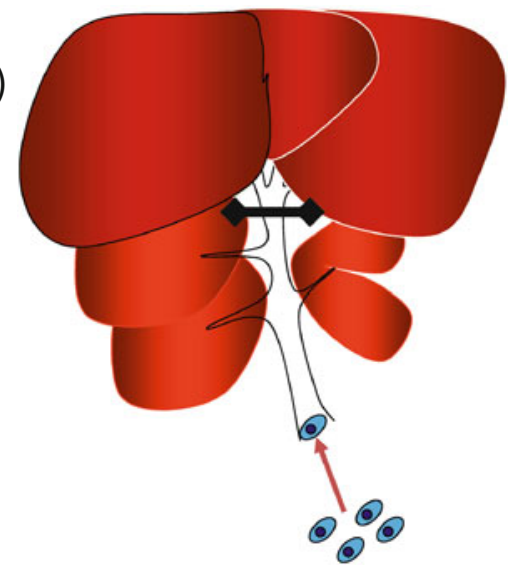

Fig. 2 Hepatectomy (70 \%) with the I/R injury model in the rat.

After 40 min of warm ischemia, $70 \%$ hepatectomy was performed, followed by injection of MSCs via the portal vein

\section{Outcome After MSC Injection}

Male Lewis rats were separated into two groups: an MSC group given MSCs after reperfusion as a treatment, and a control group given phosphate-buffered saline after reperfusion as a placebo. The results of liver function tests, pathologic changes in the liver, and the remnant liver regeneration rate were assessed. The fate of transplanted MSCs in the luciferase-expressing rats was examined by in vivo luminescent imaging. The MSC group showed peak luciferase activity of transplanted MSCs in the remnant liver $24 \mathrm{~h}$ after reperfusion, after which luciferase activity gradually declined. The elevation of serum alanine transaminase levels was significantly reduced by the MSC injection. Histopathological findings showed that the vacuolar change was lesser in the MSC group than in the control group. In addition, a significantly lower percentage of TUNEL-positive cells was observed in the MSC group than in the controls. The remnant liver regeneration rate was accelerated in the MSC group. The above results suggest that MSC transplantation provides trophic support to the I/R-injured liver by inhibiting hepatocellular apoptosis and by stimulating regeneration. 


\section{Mesenchymal Stem Cells for Pig Ischemia/Reperfusion Injury}

\section{Pig Ischemia Reperfusion Model}

Following rat hepatectomy experiments, a preclinical pig model was introduced (Fig. 3). In our model, long warm ischemia of up to $90 \mathrm{~min}$ is simulated for Pringle's procedure during hepatectomy or ischemia/reperfusion injury during liver transplantation. Inflow occlusion, such as Pringle's occlusion for a long period, causes mesenteric congestion, which affects the outcome and can sometimes be lethal. Therefore, a portacaval shunt between the splenic vein and the internal jugular vein was placed during inflow occlusion. The clamp was place at the hepatoduodenal ligament for $90 \mathrm{~min}$ with intensive monitoring for vital signs. After the declamp, MSCs $\left(1.0 \times 10^{7} / \mathrm{kg}\right)$ were injected in the MSC group. An initial problem with the MSC injection was probable hepatic embolism. Injection of the MSCs just after collection from the plates led to severe aggregation of cells, followed by embolus formation. Accordingly, we modified our protocol by immersing the MSCs in an ET-Kyoto solution so that the cells would not aggregate. Thereafter,

\section{Warm ischemia and reperfusion model (pig)}
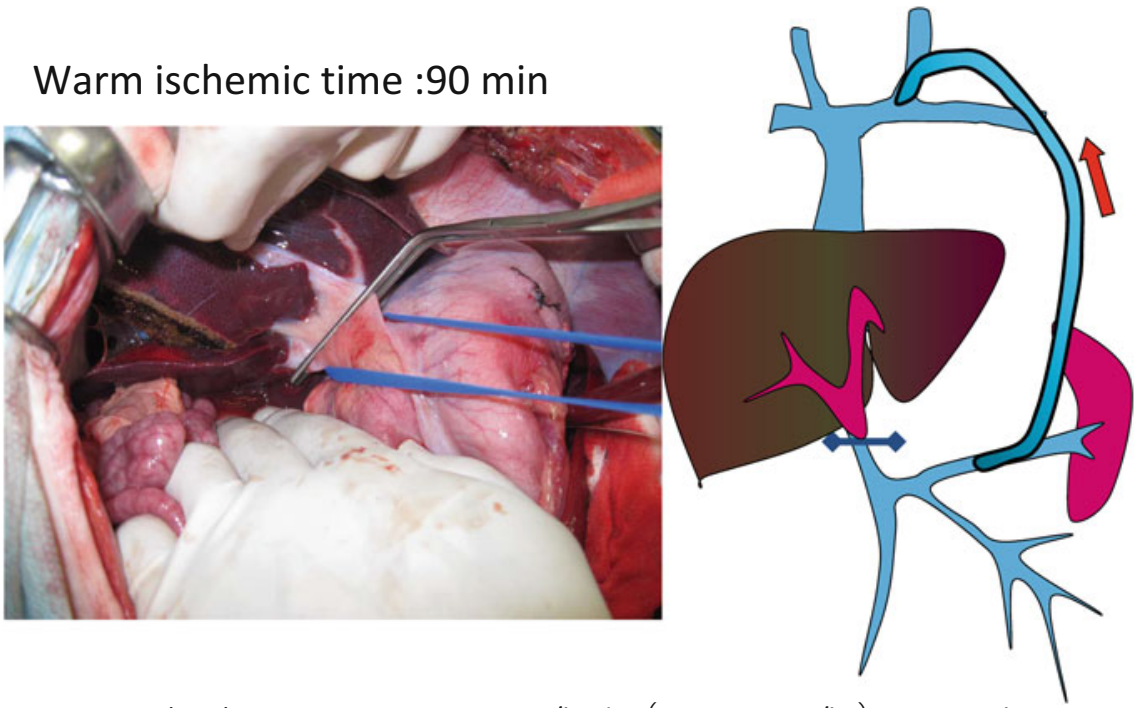

MSC group $(n=3)$ : AT-MSC $2.3 \pm 0.2 \times 10^{8} /$ body $\left(1.0 \pm 0.1 \times 10^{7} / \mathrm{kg}\right)$ via portal vein Control group $(n=3)$ : saline

Fig. 3 Warm ischemia and reperfusion model in the pig.

After 90 min of warm ischemia using Pringle's procedure, MSCs were injected via the portal vein. During Pringle's procedure, a portacaval shunt was placed in order to avoid mesenteric congestion 
no lethal complication was encountered. After the procedure, the pig was monitored for survival and daily laboratory examinations including AST, ALT, LDH, and portal venous pressure.

\section{Outcome After MSC Injection (Fig. 4)}

All pigs in the MSC group ( $n=5)$ and control group $(n=5)$ survived during an observation period for 7 days. In the control group, AST, ALT, and LDH peaked several hours after the operation and returned to normal in 3 days. However, this increase in laboratory parameters was not observed in the MSC group. In terms of adverse effects, portal venous pressure (PVP) was kept stable without an increase throughout the course, in both the MSC group and the control group. Therefore, the modified method for cell preparation is effective to avoid cell aggregation and leads to safe application of this method in the clinical setting. As we see from the laboratory results, the protective effect of MSCs is suggested in prolonged warm ischemia during surgery. This may lead to wider indications in liver surgery and transplantation.

\section{Liver enzymes and PVP: I/R model (pig)}
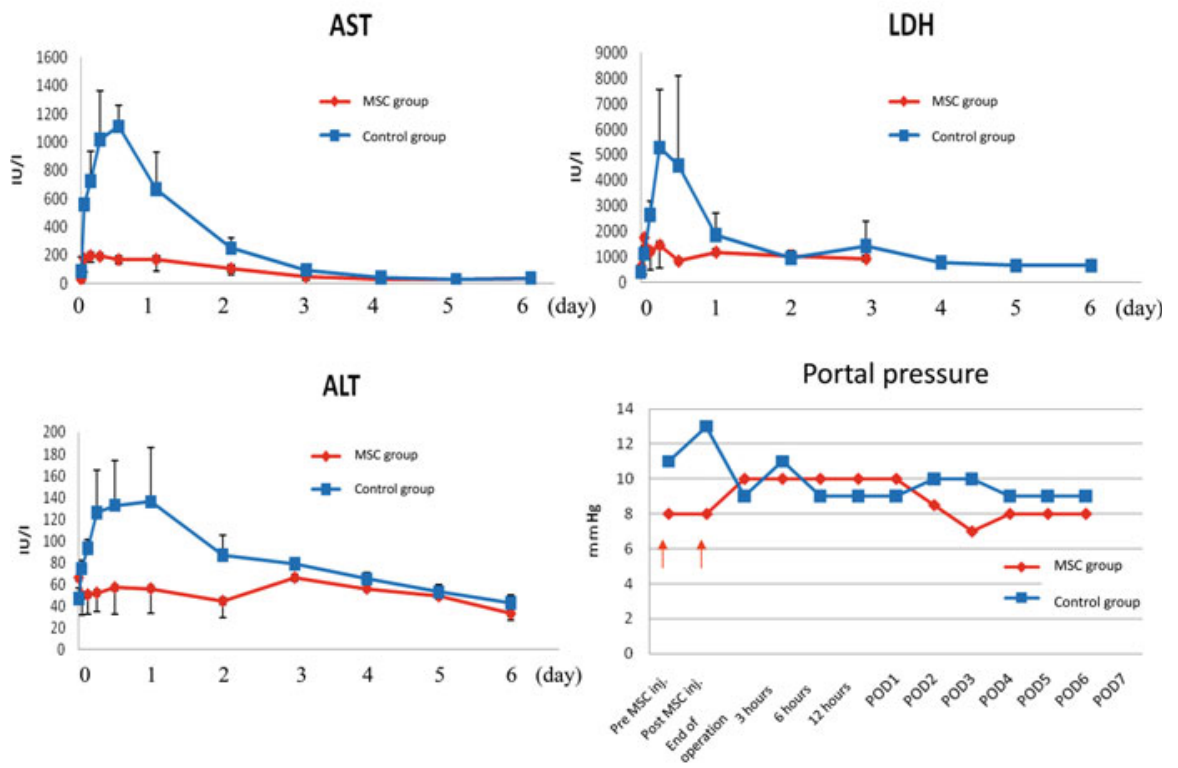

Fig. 4 Liver enzymes and PVP in the I/R model in the pig.

In the pig warm ischemia model, AST, ALT, and LDH were well controlled by MSC administration. In spite of the MSC administration, portal pressure was maintained without a significant increase 


\section{Mesenchymal Stem Cells for Rat Whole Liver Transplantation with a Long Preservation Period}

\section{Rat Liver Transplantation Model}

In the previous section, we showed that MSCs provide a protective effect against hepatic ischemia- reperfusion injury and stimulate liver regeneration in rat hepatectomy. We further evaluated the effect of MSCs on liver transplantation after prolonged cold preservation by using a rat liver transplantation model. Adipose tissue-derived MSCs from luciferase-transgenic Lewis (Luc-Tg LEW) rats were cultured and preserved in ET-Kyoto solution before use. Male Lewis rats were used as transplantation donors and recipients. The rats were randomly assigned to two groups: an MSC group and a control group. The liver grafts were retrieved and preserved in UW solution at $4{ }^{\circ} \mathrm{C}$ for $24 \mathrm{~h}$. After preservation, $1 \times 10^{5}$ of MSCs in $0.2 \mathrm{ml}$ of ET-Kyoto solution were transfused via the portal vein of the graft in the MSC group, and ET-Kyoto solution only was transfused in the control group. Then the liver graft was orthotopically transplanted into a recipient rat by the cuff technique of Kamada and Calne, with minor modification. Both the superior and inferior hepatic vena cava were anastomosed by a running suture. The portal vein was connected by a polyethylene cuff and the bile duct by a stent. The hepatic artery was reconstructed by the sleeve method. The survival of the recipient rats was evaluated for 7 days after transplantation.

\section{Outcome After MSCs Injection}

The anhepatic times were $15.5 \pm 0.7 \mathrm{~min}$ in the MSC group and $15.4 \pm 1.3 \mathrm{~min}$ in the control group. The inferior vena cava clamping times were $27.6 \pm 1.2 \mathrm{~min}$ and $27.1 \pm 1.7 \mathrm{~min}$, respectively. Both parameters did not differ significantly between the groups. Liver enzymes were better in the MSC group. In the control group, prominent necrosis and ballooning of cells in the adjacent area were observed, whereas the findings were almost normal in the MSC group (Fig. 5). In the MSC group, DLK/OV6 double positive cells were observed, whereas positive cells were minimal in the control group. These data support the positive effect of MSCs in ameliorating ischemia/reperfusion injury. The regenerative effect of MSCs was shown as well. The 7-day survival was significantly better in the MSC group than in the control group (7/8 vs. 3/8; Fisher's exact test, $p<0.05$ ) (Fig. 6). Portal transfusion of the MSCs ameliorated the injury of the liver graft after prolonged cold preservation and transplantation. 


\section{Histology: LTx with long preservation model (rat)}

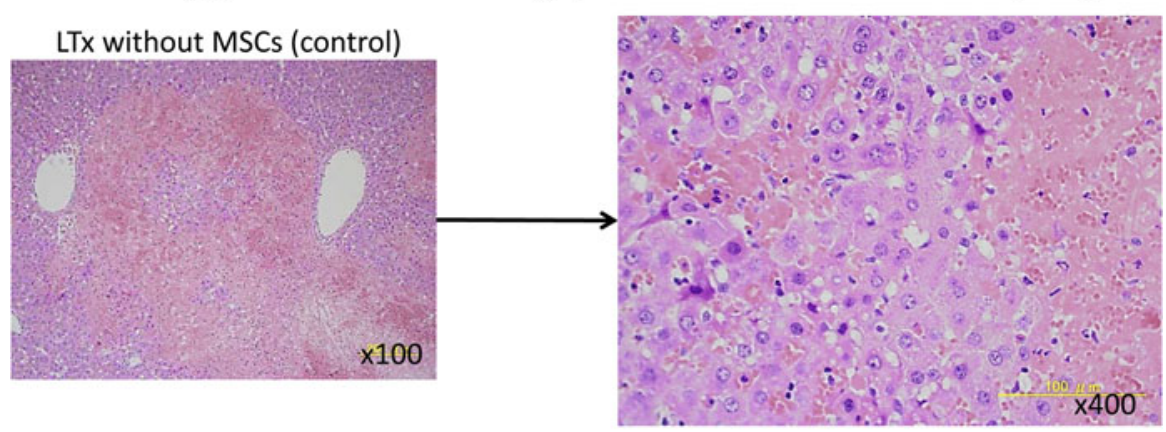

LTx with MSCs
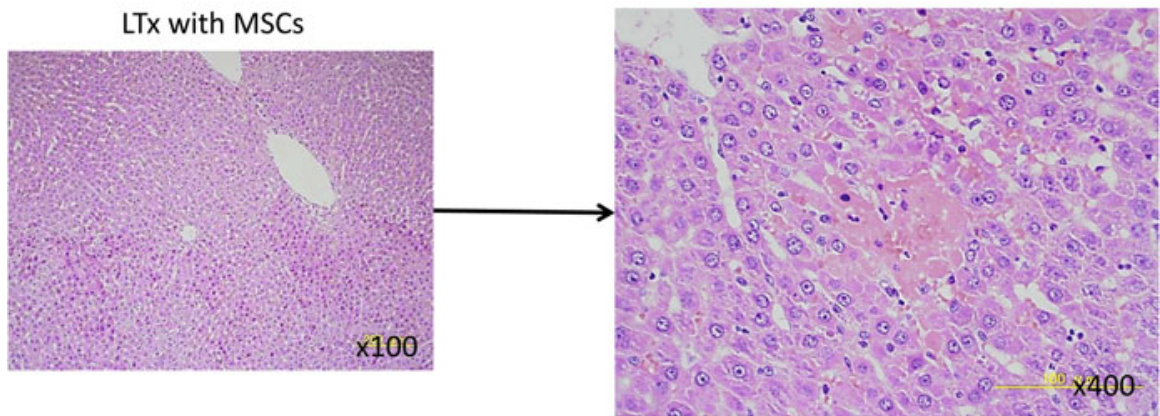

Fig. 5 Histology in liver transplantation using the long-preservation model in the rat.

An almost normal structure with minimal necrosis was maintained in the MSC group, whereas significant necrosis and hepatocyte ballooning in the adjacent area were observed in the control group

\section{Experimental Models for Clinical Application}

\section{Reduced Liver Transplantation (Rat) as a Model for Living- Donor Liver Transplantation}

During the donor operation, the right lobe (RL) of the liver was selected as the graft for the $20 \%$ PLTx group, while the superior right lobe (SRL) was selected for the $15 \%$ PLTx group, on the basis of the rat liver anatomy. Liver grafts were preserved in ice-cold HTK solution before implantation for $180 \mathrm{~min}$. The SHVC was sewn with an 8-0 Prolene continuous suture, and the portal vein was anastomosed using a cuff technique. After confirming reflow, the infrahepatic vena cava (IHVC) was anastomosed using an 8-0 nylon continuous suture. The hepatic artery was connected using a sleeve technique, and the bile duct was reconstructed over an 


\section{Survival after liver transplantation (rat)}

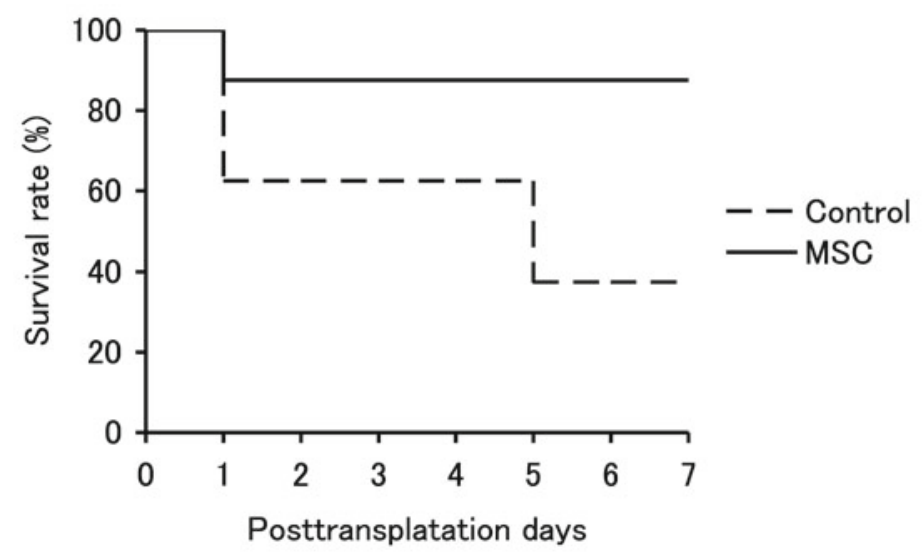

$7 / 8$ vs. $3 / 8$, MSC vs. control; $p<0.05$

Fig. 6 Survival after liver transplantation in the rat.

After 24-h preservation, only $38 \%$ (3/8) achieved long-term survival. In contrast, with administration of MSCs, $88 \%(7 / 8)$ survived at 7 days after transplantation

intraluminal stent. With this model, 7-day survival was $100 \%$ with the $20 \%$ graft and $17 \%$ with the $15 \%$ graft. Experiments assessing the efficacy of MSCs are under evaluation.

\section{Partial Liver Transplantation (Pig) as a Model for Living-Donor Liver Transplantation}

For this preclinical study, the Clawn miniature pig was used for the partial liver transplantation model [6]. The porcine liver consists of four lobes. The right lateral lobe (RLL) accounts for $30 \%$ of the whole liver. The structure of the porta hepatis was carefully dissected and cut in order to resect the lobes other than the RLL. Seventy percent of the liver was resected in the donor, not on the back table, to confirm hemostasis. Then the graft was preserved in the preservation solution for 3-24 h, followed by implantation into the recipient. In the recipient, prior to total hepatectomy, a portacaval shunt was placed to avoid mesenteric congestion. The inferior vena cava (suprahepatic and infrahepatic), portal vein, hepatic artery, and bile duct were sewn (Fig. 7). 


\section{Recipient Operation with reduced graft (pig)}

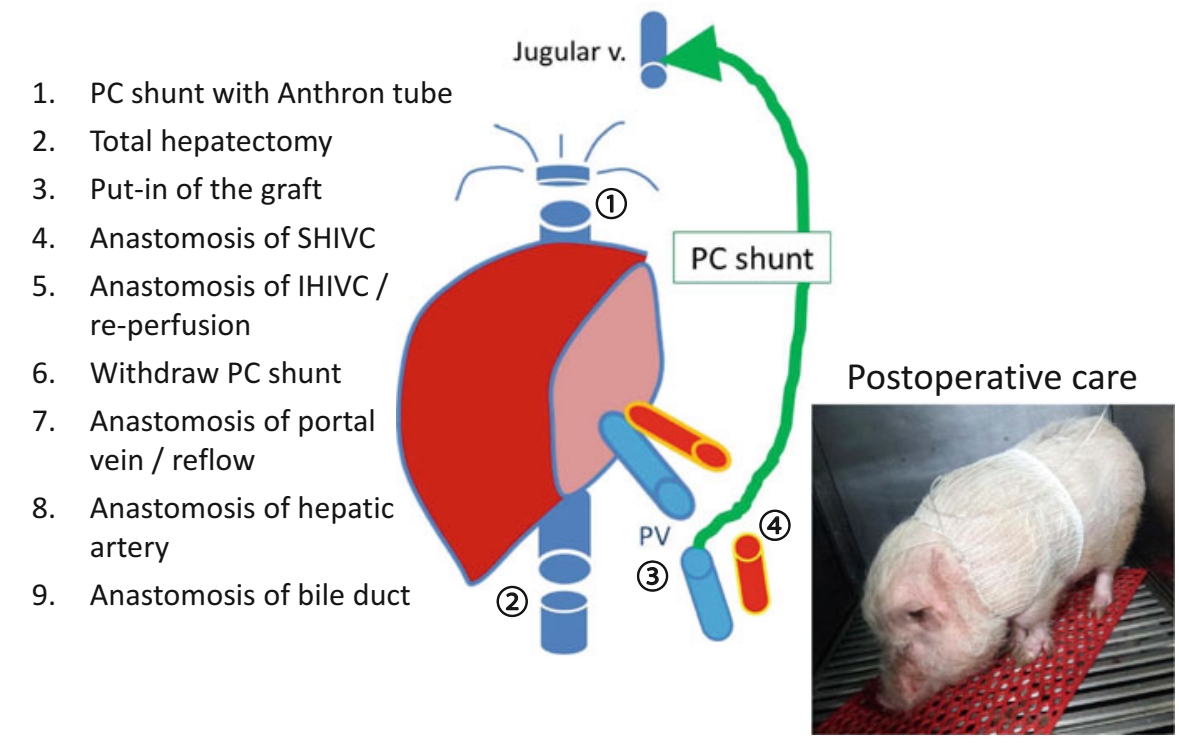

Fig. 7 Recipient operation with a reduced graft in the pig.

In order to avoid mesenteric congestion, a portacaval shunt was placed prior to total hepatectomy. Following total hepatectomy, implantation of the graft was done with multiple anastomoses

\section{Future Perspectives}

\section{Source for MSCs}

MSC have been isolated from almost every type of tissue stroma, such as the bone marrow, adipose tissue, umbilical cord blood, placenta, kidney, liver, heart, and spleen. There are many reports on the positive immunomodulatory effect or regenerative effect of MSCs of bone marrow origin. However, retrieval of bone marrow from healthy volunteers or patients seems invasive. On the other hand, Banas et al. reported a stronger effect of adipose tissue-derived MSCs compared with BM-MSCs. Moreover, subcutaneous adipose tissue can be safely retrieved under local anesthesia or during a major operation. We have been focusing on utilizing MSCs from adipose tissue. The remaining problem is from whom and when adipose tissue can be retrieved. Potential donors are the patient him- or herself, the donor of the organ in transplantation, or a third party. One of the advantages of MSCs compared with other cell sources is that MSCs express less HLA, leading to less rejection. Moreover, the regenerative or immunomodulatory effects are expected only in the short term, not in the long term. For use in cancer patients, we propose use of the patient's own MSCs without immunosuppression. On the other hand, cells from all 
three sources can be utilized in transplant recipients under immunosuppression. For an elective cancer operation, the patient's own MSCs can be retrieved and cultured for several weeks. However, stocked cells from a third party can be used for organ transplantation performed on an emergency basis [7, 8].

\section{Complications}

The most severe complication we have encountered is embolus formation due to cell aggregation. However, with the modification using ET-Kyoto solution, we can keep cells in a suspended form for several hours, which secures safe administration of the MSCs. ET-Kyoto solution is commercially available, and its safety is established. Potential transformation of MSCs into malignant cells can be problematic; however, the lifespan of the cells is limited, according to our study, and they can be eliminated within 2 weeks. Mild rejection will help to eliminate cells after a certain period as well.

\section{Conclusion}

Thanks to the support of the Uehara Memorial Foundation, we have been able to show the positive effect of MSCs with liver surgery. Cell damage during ischemia reperfusion injury is ameliorated, and liver regeneration after hepatectomy is enhanced by use of intravenous or intraportal MSCs. These positive effects have been shown both in vitro and in vivo including in a preclinical pig model.

Open Access This chapter is distributed under the terms of the Creative Commons Attribution Noncommercial License, which permits any noncommercial use, distribution, and reproduction in any medium, provided the original author(s) and source are credited.

\section{References}

1. Uemoto S (2010) [Liver transplantation]. Nihon Rinsho 68(12):2277-2280

2. Yagi $S$ et al (2012) Small-for-size syndrome in living donor liver transplantation. Hepatobiliary Pancreat Dis Int 11(6):570-576

3. Banas A et al (2008) IFATS collection: in vivo therapeutic potential of human adipose tissue mesenchymal stem cells after transplantation into mice with liver injury. Stem Cells 26(10):2705-2712

4. Banas A et al (2007) Adipose tissue-derived mesenchymal stem cells as a source of human hepatocytes. Hepatology 46(1):219-228

5. Kanazawa $\mathrm{H}$ et al (2011) Bone marrow-derived mesenchymal stem cells ameliorate hepatic ischemia reperfusion injury in a rat model. PLoS One 6(4):e19195 
6. Hori T et al (2014) How to successfully resect $70 \%$ of the liver in pigs to model an extended hepatectomy with an insufficient remnant or liver transplantation with a small-for-size graft. Surg Today 44(11):2201-2207

7. Dahlke MH et al (2009) Toward MSC in solid organ transplantation: 2008 position paper of the MiSOT Study Group. Transplantation 88(5):614-619

8. Franquesa M et al (2013) Mesenchymal Stem Cells in Solid Organ Transplantation (MiSOT) Fourth Meeting: lessons learned from first clinical trials. Transplantation 96(3):234-238 